\title{
Existence of attractor for wave equation with decay coefficient on $\mathrm{R}^{\mathrm{n}}$
}

\section{Tingting Hu, Tingting Liu, Qiaozhen Ma}

Abstract - In this paper, by using the method called "asymptotic contractive process" on the timedependent entire space, the existence of time-dependent attractor for the wave equation with decay coefficient on $H^{1}\left(\square^{n}\right) \times L^{2}\left(\square^{n}\right)$ is obtained.

Key words - wave equation, time-dependent attractor, asymptotic contractive process, un-bounded domain. AMS Subject Classification: 35B25;37L30;45K05.

\section{Introduction}

We onsider the asymptotic behavior of solutions to the following equations on $\square^{3}$ :

$$
\left\{\begin{array}{l}
\varepsilon(t) u_{t t}+\alpha u_{t}-\Delta u+\lambda u+f(x, u)=g(x), x \in \square^{3}, t \geq \tau, \\
u(x, \tau)=u_{0}(x), u_{t}(x, \tau)=u_{1}(x), x \in \square^{3},
\end{array}\right.
$$

where $u(x, t)$ is an unknown function, $\lambda>0, \varepsilon=\varepsilon(t)$ is a decreasing bounded function and

$$
\lim _{t \rightarrow+\infty} \varepsilon(t)=0
$$

Especially, there exists a constant $L>0$ such that

$$
\sup _{t \in \square}\left[|\varepsilon(t)|+\left|\varepsilon^{\prime}(t)\right|\right] \leq L .
$$

The nonlinear term $f(x, s) \in C\left(\square^{4}\right)$ with $f(x, \cdot) \in C^{2}(\square)$ for every fixed $x \in \square^{3}$, and satisfies

$$
\begin{gathered}
f(\cdot, 0) \in L^{2}\left(\square^{3}\right),\left|\frac{\partial f}{\partial s}(x, 0)\right| \leq c_{0}, \forall x \in \square^{3}, \\
\left|\frac{\partial f}{\partial s}(x, \cdot)\right| \leq c_{1}\left(1+|s|^{2}\right),\left|\frac{\partial f}{\partial x}(\cdot, s)\right| \leq c_{2}, \forall s \in \square, x \in \square^{3}, \\
\liminf _{|s| \rightarrow \infty} \frac{\partial f(x, s)}{\partial s}>-\lambda_{1}, \forall s \in \square, x \in \square^{3}, \\
(f(x, s)-f(x, 0)) \geq c_{3} s^{2}, \forall s \in \square,|x| \geq r_{0}>0,
\end{gathered}
$$

where $c_{i}>0, i=0,1,2,3, \lambda_{1}$ is the first eigenvalue of the operator $A=-\Delta$.

Equation (1.1) can be seen as a nonlinear damped wave equation with time-dependent speed of

Tingting Hu, School of Mathematics and Statistics, Northwest Normal University, Lanzhou, Gansu, China, 18093364636.

Tingting Liu, School of Mathematics and Statistics, Northwest Normal University, Lanzhou, Gansu, China, 18093364636.

Qiaozhen Ma, School of Mathematics and Statistics, Northwest Normal University, Lanzhou, Gansu, China, 13659416525. propagation $\frac{1}{\varepsilon(t)}$. Besides, it can also be regarded as a model for the thermal evolution in ahomogeneous isotropic (rigid) heat conductor according to the Maxwell-Cattaneo law [8] (see also [12, AppendixB]), with $\varepsilon(t)$ representing a time-dependent relaxation parameter.

When $\varepsilon$ is only a positive constant in (1.1), the asymptotic behavior of solutions to equation (1.1) on bounded domains has been the object of extensive studies since the eighties (see,e.g. [8-16]).

For equation (1.1), in [1], Conti, Pata and Temam presented a notion of time-dependent attractor exploiting the minimality with respect to the pullback attraction property, and constructed a sufficient condition proving the existence of time-dependent attractor based on the theory established by Plinio, Duane and Temam([5]). Meanwhile, within the new framework, on bounded domain, the authors studied the following weak damped wave equations with time-dependent speed of propagation

$$
\varepsilon(t) u_{t t}+\alpha u_{t}-\Delta u+f(u)=g(x), x \in \Omega \subset \square^{3},
$$

in particular, they proved that the time-dependent global attractor of (1.8) converged in a suitable sense to the attractor of the parabolic equation $\alpha u_{t}-\Delta u+f(u)=g(x)$ when $\varepsilon(t) \rightarrow 0$ as $t \rightarrow+\infty$ ([2]). Successively, in[3], they continued to show the existence of an invariant time-dependent global attractor to the following specific one-dimensional wave equation $\varepsilon(t) u_{t t}-u_{x x}+\left[1+\varepsilon f^{\prime}(u)\right] u_{t}+f(u)=h$, which converges in suitable sense to the classical Fourier equation.

Recently, Meng et al. investigated the long-time behavior of the solution for the wave equation with nonlinear damping $g\left(u_{t}\right)$ on the time-dependent space, in which they found a new technical method verifying compactness of the process via defining the contractive functions, see [6]. In [7], Meng and Liu also showed the necessary and sufficient conditions of the existence of time-dependent global attractor borrowed from the ideas in [17]. 
However, all researches about the time-dependent attractor were on bounded domain. In the recent, the authors gave a method called"asymptotic contractive process"to prove that the process is pullback asymptotic compactness on unbounded domian ([18]). So in this paper, we exploiting this new method to show the existence of time-dependent attractor for (1.1) on unbounded domain.

The rest of this article consists of four Sections. In the next Section, we define some functions sets and iterate some useful lemmas; in Section 3, we introduce the concept of asymptotic contractive process and a technique for verifying asymptotic compactness for the process is proposed; finally, dissipativity, tail estimate and the existence of the time-dependent global attractor is obtained on $\square^{3}$ in Section 4.

\section{Preliminaries}

Now we recall some basic notations and abstract results in[1,6,18], which are necessary for getting our main results.

\subsection{Notations.}

Without loss of generality, set $\mathrm{H}=L^{2}\left(\square^{n}\right)$, endowed with the inner product $\langle\cdot, \cdot\rangle$ and norm $\|$, respectively. For $0 \leq s \leq 2$, we define the hierarchy of nested Hilbert spaces

$$
\mathrm{H}^{s}=\mathrm{H}^{s}\left(\square^{n}\right)=\mathrm{D}\left(A^{\frac{s}{2}}\right),\langle w, v\rangle_{s}=\left\langle A^{\frac{s}{2}} w, A^{\frac{s}{2}} v\right\rangle\|\|\|\|_{s}^{2}=\left\langle A^{\frac{s}{2}} w, A^{\frac{s}{2}} w\right\rangle .
$$

Now, for $t \in \square$ and $0 \leq s \leq 2$, write the following symbols,

with the norm

$$
\mathrm{H}_{t}^{s}=\mathrm{H}^{s+1} \times \mathrm{H}^{s},
$$

$$
\|z\|_{\mathrm{H}_{t}^{s}}^{2} \#\left(u, u_{t}\right)\left\|_{\mathrm{H}_{t}^{s}}^{2} \# u\right\|_{s+1}^{2}+\varepsilon(t)\left\|u_{t}\right\|_{s}^{2} .
$$

The letter s is always omitted whenever zero. Especially, we consider the time-dependent phase space

$$
\mathrm{H}_{t}=\mathrm{H}^{1} \times \mathrm{H} \text {, }
$$

with the norm

$$
\|z\|{ }_{\mathrm{H}_{t}}^{2} \#\left(u, u_{t}\right)\left\|_{\mathrm{H}_{t}}^{2} \# u\right\|_{2}^{2}+\varepsilon(t)\left\|u_{t}\right\|^{2} \# \nabla u\left\|^{2}+\right\| u\left\|^{2}+\varepsilon(t)\right\| u_{t} \|^{2} .
$$

For every $t \in \square$, let $X_{t}$ be a family of normed spaces, we introduce the $\rho$-ball of $X_{t}$

$$
\mathrm{B}_{t}(\rho)=\left\{z \in X_{t}\|z\|_{X_{t}} \leq \rho\right\} .
$$

We denote the Hausdorff semi-distance of two (nonempty) sets $B, C \in X_{t}$ by

$$
\delta_{t}(B, C)=\sup _{x \in B} \operatorname{dist}_{X_{t}}(x, C)=\operatorname{supinf}_{x \in B}\|x-y\|_{X_{t}} .
$$

For any given ò $>0$, the ò-neighbourhood of a set $B \subset X_{t}$ is defined as

$$
\mathrm{O}_{t}^{\grave{\mathrm{o}}}(B)=\bigcup_{x \in B}\left\{y \in X_{t}\|y-x\|_{X_{t}}<\mathrm{o}\right\}=\bigcup_{x \in B}\left\{x+\mathrm{B}_{t}(\grave{\mathrm{o}})\right\} .
$$

2.2 Some concepts and abstract results.

Definition 2.1. ([1]). Let $\left\{X_{t}\right\}_{t \in \square}>0$ be a family of normed spaces. A process is a two-parameter family of mappings $\left\{U(t, \tau): X_{\tau} \rightarrow X_{t}, t \geq \tau \in \square\right\}$ with properties (i) $U(\tau, \tau)=I d$ is the identity on $X_{\tau}, \tau \in \square$; (ii) $U(t, s) U(s, \tau)=U(t, \tau), \forall t \geq s \geq \tau$

Definition 2.2. ([1]). A family $\mathrm{C}=\left\{C_{t}\right\}_{t \in \square}$ of bounded sets $C_{t} \subset X_{t}$ is called uniformly bounded if there exists a constant $\rho>0$ such that $C_{t} \subset \mathrm{B}_{t}(\rho), \forall t \in \square$.

Definition 2.4. ([1]). A (uniformly bounded) family $\mathrm{K}=\left\{K_{t}\right\}_{t \in \square}$ is called pullback attracting if for all $\mathrm{o}>0$, the family $\left\{\mathrm{O}_{t}^{\dot{o}}\left(K_{t}\right)\right\}_{t \in \square}$ is pullback absorbing.

Definition 2.5. ([1]). The time-dependent global attractor for $U(t, \tau)$ is the smallest family $\mathrm{A}=\left\{A_{t}\right\}_{t \in \square}$ with the following properties:

(i) Each $A_{t}$ compact in $X_{t}$;

(ii) A is pullback attracting, namely, it is uniformly bounded and the limit

$$
\lim _{\tau \rightarrow-\infty} \delta_{t}\left(U(t, \tau) C_{\tau}, A_{t}\right)=0
$$

holds for every uniformly bounded family $\mathrm{C}=\left\{C_{t}\right\}_{t \in \square}$ and every $t \in \square$.

Theorem 2.6. ([1]). The time-dependent attractor $\mathrm{A}$ exists and it is unique if and only if the process $U(t, \tau)$ is asymptotically compact, namely, the set

$\mathrm{K}=\left\{\mathrm{K}=\left\{K_{t}\right\}_{t \in \square}: K_{t} \subset X_{t}\right.$ compact, Kpullbackattracting $\} \neq \varnothing$.

Definition 2.7. ([17]). Let $\left\{X_{t}\right\}_{t \in \square}$ be a Banach space and $\mathrm{C}=\left\{C_{t}\right\}_{t \in \square}$ be a family of uniformly bounded subset of $\left\{X_{t}\right\}_{t \in \square}$. We call a function $\psi_{\tau}^{t}(\cdot, \cdot)$ defined on $\left\{X_{t}\right\}_{t \in \square} \times\left\{X_{t}\right\}_{t \in \square}$ to be a asymptotic contractive function on $C_{t} \times C_{t}$, if for any $t \in \square$ and any sequence $\left\{x_{n}\right\}_{n=1}^{\infty} \subset \mathrm{C}_{t}$, such that for any $\mathrm{o}>0$, there is a subsequence $\left\{x_{n_{k}}\right\}_{k=1}^{\infty} \subset\left\{x_{n}\right\}_{n=1}^{\infty}$ satisfying:

$$
\psi_{\tau}^{t}\left(x_{n_{k}}, x_{n_{l}}\right) \leq \grave{\mathrm{o}}+\phi_{\tau}^{t}\left(x_{n_{k}}, x_{n_{l}}\right),
$$

where

$$
\lim _{k \rightarrow \infty} \lim _{l \rightarrow \infty} \phi_{\tau}^{t}\left(x_{n_{k}}, x_{n_{l}}\right)=0, \tau \leq t .
$$

Definition 2.8. ([17]). Let $U(\cdot, \cdot)$ be a process on $\left\{X_{t}\right\}_{t \in \square}$ and for any ̀̀ $>0$ there exist $\tau<T(\grave{\mathrm{o}}) \leq t, \psi_{T}^{t} \in \mathrm{C}\left(C_{T}\right)$, such that

$$
\|U(t, T) x-U(t, T) y\|_{X_{t}} \leq \grave{\mathrm{o}}+\psi_{T}^{t}(x, y), \forall x, y \in C_{T},
$$

for any fixed $t \in \square$. Then $U(\cdot, \cdot)$ is called asymptotic contractive process. 
Theorem 2.9. ([17]). Let $U(\cdot, \cdot)$ be a process in a family of Banach space $\left\{X_{t}\right\}_{t \in \square}$. Then $U(\cdot, \cdot)$ has a time-dependent global attractor $\mathrm{U}^{*}=\left\{\mathrm{A}_{t}^{*}\right\}_{t \in \square}$ in $\left\{X_{t}\right\}_{t \in \square}$ provided that the following conditions hold true:

(i) $U(\cdot, \cdot)$ has a pullback absorbing family $\mathrm{B}=\left\{B_{t}\right\}_{t \in \square}$;

(ii) $U(\cdot, \cdot)$ is pullback asymptotic contractive process on $B_{t}$. Let

$$
F(x, u)=\int_{0}^{u} f(x, y) d y .
$$

Lemma 2.10. ([10,11]). From (1.4), (1.6)-(1.7), for $0<v<\min \{1, \lambda\}$, there exist $\tilde{\mathrm{n}}(v)>0, c(v) \geq 0(i=1,2)$, such that the following inequalities hold for every $u \in \mathrm{H}^{1}$ :

$$
\begin{gathered}
2\langle F(x, u), 1\rangle \geq-v\|u\|^{2}-c(v), \\
\langle f(x, u), u\rangle+\frac{v_{\|}}{2} \nabla u\left\|^{2} \geq \tilde{\mathrm{n}}(v)\right\| u \|^{2}-c(v) .
\end{gathered}
$$

\section{Dissipativity}

Global existence of solution $u$ to (1.1) is classical, by using the standard Galerkin approximation method ([1, 12, $16]$ ), so we only give the following results and omit the proof.

Lemma 3.1. Under the assumptions(1.2)-(1.7), for every pair of initial data $z_{\tau} \in \mathrm{H}_{\tau}, g \in L^{2}\left(\square^{3}\right)$, there exists a unique solution $z(t)=\left(u, u_{t}\right)$ of problem (1.1) in space $\mathrm{H}_{t}$ and satisfy

$$
z \in C\left([\tau, t] ; \mathrm{H}_{t}\right) \cap L^{\infty}\left([\tau, t) ; \mathrm{H}_{t}^{1}\right) .
$$

Furthermore, let $z_{i}(\tau) \in \mathrm{H}_{\tau}$ be the initial data such that $\left\|z_{i}(\tau)\right\|_{\mathrm{H}_{\tau}} \leq \rho(i=1,2)$, and $z_{i}(t)$ be the solution of problem (1.1). Then there exists $K=K(\rho)>0$, such that

$\left\|z_{1}(t)-z_{2}(t)\right\|_{\mathrm{H}_{t}} \leq e^{K(t-\tau)}\left\|z_{1}(\tau)-z_{2}(\tau)\right\|_{\mathrm{H}_{\tau}}, \forall \mathrm{t} \geq \tau$.

Lemma 3.2. Under the assumptions(1.2)-(1.7), $g \in L^{2}\left(\square^{3}\right)$, for any initial data $z_{\tau} \in \mathrm{B}_{\tau}\left(\rho_{0}\right) \subset \mathrm{H}_{\tau}$, there exists $\rho>0$, such that

$\|U(t, \tau) z(\tau)\|_{\mathrm{H}_{\tau}} \leq \rho, \forall \tau \leq t$.

Proof Multiplying (1.1) by $v=u_{t}+\delta u$ and integrating over $\square^{3}$, we obtain

$$
\frac{d}{d t} E(t)+I(t)=0
$$

where

$$
\begin{aligned}
& E(t) \# \nabla u\left\|^{2}+\lambda\right\| u\left\|^{2}+\varepsilon(t)\right\|\|\|^{2}+2\langle F(x, u), 1\rangle-2\langle g, u\rangle, \\
& I(t)=\left(2(\alpha-\delta \varepsilon(t))-\varepsilon^{\prime}(t)\right)\|\|^{2}+2 \delta\|\nabla u\|^{2}+2 \delta \lambda\|u\|^{2} \\
& -2 \delta(\alpha-\delta \varepsilon(t))\langle u, v\rangle+2 \delta\langle f(x, u), u\rangle-2 \delta\langle g, u\rangle,
\end{aligned}
$$

integrating (3.2) over $[\tau, t]$, there holds

$$
E(t)=-\int_{\tau}^{t} I(s) d s+E(\tau)
$$

Let $0<\delta<\min \left\{\frac{\alpha}{6 L}, \frac{\lambda+4 \tilde{n}(v)}{2 \alpha}\right\}$, such that

$$
\frac{\alpha}{2}-2 \delta \varepsilon(t)>\delta \varepsilon(t), \frac{3}{2} \delta \lambda+2 \delta \tilde{\mathrm{n}}(v)-\delta^{2} \alpha>\delta \lambda,
$$

then by (2.2), Hölder, Young inequalities, we obtain

$$
\begin{aligned}
& I(t) \geq \delta(2-v)\|\nabla u\|^{2}+2 \delta(\lambda+\tilde{\mathrm{n}}(v))\|u\|^{2}+(2 \alpha-2 \delta \varepsilon(t))\|v\|^{2} \\
& -\left(\alpha\|v\|^{2}+\delta^{2} \alpha\|u\|^{2}\right)-\left(\frac{\delta \lambda}{2}\|u\|^{2}+\frac{2 \delta}{\lambda}\|g\|^{2}\right)-2 \delta c_{2} \\
& \geq \delta v\|\nabla u\|^{2}+\left(\frac{3}{2} \delta \lambda+2 \delta \tilde{n}(v)-\delta^{2} \alpha\right)\|u\|^{2}+(\alpha-2 \delta \varepsilon(t))\|v\|^{2} \\
& -\frac{2 \delta\|g\|^{2}-2 \delta c_{2}}{\lambda} \\
& \left.\geq \delta v\|\nabla u\|^{2}+\|u\|^{2}+\varepsilon(t)\|v\|^{2}\right)+\frac{\alpha}{2}\|\|^{2}-\frac{2 \delta}{\lambda}\|g\|^{2}-2 \delta c_{2},
\end{aligned}
$$

combining with (2.2) there holds

$$
\begin{aligned}
& E(t) \# \nabla u\left\|^{2}+\left(\frac{3}{4} \lambda-v\right)\right\| u\left\|^{2}+\varepsilon(t)\right\| v \|^{2}-\left(\frac{4}{\lambda}\|g\|^{2}+c_{1}\right) \\
& \left.\geq \frac{v}{2}\|\nabla u\|^{2}+\|u\|^{2}+\varepsilon(t)\|v\|^{2}\right)-\left(\frac{4}{\lambda}\|g\|^{2}+c_{1}\right),
\end{aligned}
$$

Together with (3.3), (3.4)-(3.5) it follows that

$$
\begin{aligned}
& \frac{v}{2}\left(\|\nabla u\|^{2}+\|u\|^{2}+\varepsilon(t)\|v\|^{2}\right)-m_{1} \\
& \left.\leq-\int_{\tau}^{t}\left[\delta v\|\nabla u\|^{2}+\|u\|^{2}+\varepsilon(t)\|v\|^{2}\right)-m_{2}\right] d r+E(\tau),
\end{aligned}
$$

where $m_{1}=\left(\frac{4}{\lambda}\|\|^{2}+c_{1}\right), m_{2}=\frac{2 \delta}{\lambda}\|g\|^{2}+2 \delta c_{2}$. So, for any $\rho_{0}>\frac{m_{2}}{\delta v}$, there exists $t_{0}>\tau$ such that

$$
\left\|\nabla u\left(t_{0}\right)\right\|^{2}+\left\|u\left(t_{0}\right)\right\|^{2}+\varepsilon\left(t_{0}\right)\left\|v\left(t_{0}\right)\right\|^{2} \leq \rho_{0} .
$$

As a result, let $B_{t}=\bigcup_{t \geq \tau} U(t, \tau) B_{0}$, where

$$
B_{0}=\left\{\left(u_{0}, u_{1}\right) \in \mathrm{H}_{\tau}\left\|u_{0}\right\|_{1}^{2}+\varepsilon(\tau)\left\|u_{1}\right\|^{2} \leq \rho_{0}\right\},
$$

then $B_{t}$ is a bounded absorbing set for process $\{U(t, \tau)\}$.

On the other hand, from the above discussion, there exist a positive constant $\rho$ such that

$\|u\|_{1}^{2}+\varepsilon(t)\left\|u_{t}\right\|^{2} \leq \rho, \forall t \geq t_{0}>\tau$.

From Lemma 4.2, we can get the following conclusion:

Lemma 3.3. Under the assumptions(1.2)-(1.7), for $\rho_{1}>0$ in Lemma 4.2, such that $\mathrm{B}=\left\{\mathrm{B}_{t}\left(\rho_{1}\right)\right\}$ is a time-dependent absorbing sets for the process $\{U(t, \tau)\}$ associated with (1.1) then for some $R_{0} \geq \rho_{0}$, there have

$$
\left.\sup _{z \in \mathrm{B}_{\tau}\left(\rho_{1}\right)}\|U(t, \tau) z(\tau)\|_{\mathrm{H}_{t}}+\int_{\tau}^{\infty}\|v(y)\|^{2} d y\right\} \leq R_{0}, \forall t \in \square
$$

Proof Combining with (3.2), (3.4) with $\delta=0$, we get 


$$
\frac{d}{d t} E(t)+\frac{\alpha}{2}\|\|^{2} \leq \frac{2 \delta}{\lambda}\|g\|^{2}+2 \delta c_{2},
$$

integrate on $[\tau,+\infty)$, we can easily conclude that (3.7) is true.

Lemma 3.4. Under the assumptions (1.2)-(1.7), $g \in H^{1}\left(\square^{3}\right)$, for any initial data $z(\tau) \in \mathrm{B}_{\tau}\left(R_{0}\right) \subset \mathrm{H}_{\tau}^{1}$, there exists $\rho_{2}>0$, such that

$$
\varepsilon(t)\left\|A^{\frac{1}{2}} v\right\|^{2}+\|A u\|^{2} \leq \rho_{1}, \forall \tau \leq t .
$$

Proof Multiplying (1.1) by $A v=A u_{t}+\delta A u$ and integrating over $\square^{n}$, we obtain

$$
\begin{aligned}
& \frac{d}{d t}\left(\varepsilon(t)\left\|A^{\frac{1}{2}} v\right\|^{2}+\|A u\|^{2}+\lambda\left\|A^{\frac{1}{2}} u\right\|^{2}\right)-\varepsilon^{\prime}(t)\left\|A^{\frac{1}{2}} v\right\|^{2} \\
& +2(\alpha-\delta \varepsilon(t))\left\|A^{\frac{1}{2}} v\right\|^{2}-2 \delta(\alpha-\delta \varepsilon(t))\langle u, A v\rangle+2 \delta\|A u\|^{2} \\
& +2 \delta \lambda\left\|A^{\frac{1}{2}} u\right\|^{2}+2\langle f(x, u), A v\rangle=2\langle g, A v\rangle .
\end{aligned}
$$

By (3.8) Hölder, Young inequalities

$2|\langle f(x, u), A v\rangle|=2\left\langle\frac{\partial f}{\partial x}, A^{\frac{1}{2}} v\right\rangle+2\left\langle\frac{\partial f}{\partial u}, A^{\frac{1}{2}} v\right\rangle$

$\leq 2\left|f^{\prime}(u)\right|\left\|A^{\frac{1}{2}} u\right\|\left\|A^{\frac{1}{2}}\right\|\left\|\leq \frac{\alpha}{4}\right\| A^{\frac{1}{2}} u\left\|+\frac{4 l^{2}}{\alpha}\right\| A^{\frac{1}{2}} u \|^{2}$,

$2|\langle g, A v\rangle| \leq 2\|g(x)\|_{H^{1}\left(\square^{3}\right)}\left\|A^{\frac{1}{2}} v\right\| \leq \frac{\alpha}{4}\left\|A^{\frac{1}{2}} v\right\|^{2}+\frac{4}{\alpha}\|g(x)\|_{H^{1}\left(\square^{3}\right)}^{2}$,

and

$$
\begin{aligned}
& 2 \delta\|A u\|^{2}+(2 \alpha-2 \delta \varepsilon(t))\left\|A^{\frac{1}{2}} v\right\|^{2}-\varepsilon^{\prime}(t)\left\|A^{\frac{1}{2}} v\right\|^{2}- \\
& 2 \delta(\alpha-\delta \varepsilon(t))\left\langle u, A^{\frac{1}{2}} v\right\rangle \\
& \geq 2 \delta\|A u\|^{2}+(2 \alpha-2 \delta \varepsilon(t))\left\|A^{\frac{1}{2}} v\right\|^{2}-\left[\frac{\alpha}{2}\left\|A^{\frac{1}{2}} v\right\|^{2}+2 \delta^{2} \alpha\left\|A^{\frac{1}{2}} u\right\|^{2}\right] \\
& \geq 2 \delta\|A u\|^{2}+\left(\frac{3}{2} \alpha-2 \delta \varepsilon(t)\right)\left\|A^{\frac{1}{2}} v\right\|^{2}-2 \delta^{2} \alpha\left\|A^{\frac{1}{2}} u\right\|^{2} \\
& \left.\geq \delta\|A u\|^{2}+\varepsilon(t)\left\|A^{\frac{1}{2}} v\right\|^{2}\right)+\alpha\left\|A^{\frac{1}{2}} v\right\|^{2}-2 \delta^{2} \alpha\left\|A^{\frac{1}{2}} u\right\|^{2} .
\end{aligned}
$$

Combining with the above inequality, we get

$$
\begin{aligned}
& \frac{d}{d t}\left[\varepsilon(t)\left\|A^{\frac{1}{2}} v\right\|^{2}+\|A u\|^{2}+\lambda\left\|A^{\frac{1}{2}} u\right\|^{2}\right]+\delta\left(\varepsilon(t)\left\|A^{\frac{1}{2}} v\right\|^{2}\right. \\
& \left.+\|A u\|^{2}+\lambda\left\|A^{\frac{1}{2}} u\right\|^{2}\right)+\frac{\alpha}{2}\left\|A^{\frac{1}{2}} v\right\|^{2} \\
& \leq \frac{4}{\alpha}\|g\|_{H^{1}\left(\square^{3}\right)}^{2}+\left(\frac{4 l^{2}}{\alpha}+2 \delta^{2} \alpha\right)\left\|A^{\frac{1}{2}} u\right\|^{2},
\end{aligned}
$$

applying the Gronwall Lemma to (3.10) over $[\tau, t]$ and combining with the above equality we ha

$$
\begin{aligned}
& \varepsilon(t)\left\|A^{\frac{1}{2}} v\right\|^{2}+\|A u\|^{2}+\lambda\left\|A^{\frac{1}{2}} u\right\|^{2} \\
& \leq e^{-\delta(t-\tau)}\left[\varepsilon(t)\left\|A^{\frac{1}{2}} v_{\tau}\right\|^{2}+\left\|u_{0}\right\|^{2}+\lambda\left\|A^{\frac{1}{2}} u_{0}\right\|^{2}\right]+ \\
& \left(\frac{4}{\alpha} g \|_{H^{1}\left(\square^{3}\right)}^{2}+\left(\frac{4 l^{2}}{\alpha}+2 \delta^{2} \alpha\right) \cdot R_{0}^{2}\right) \\
& \leq \rho_{1},
\end{aligned}
$$

where $\rho_{1}=2\left(\frac{4}{\alpha}\|g\|_{H^{1}\left(\square^{3}\right)}^{2}+\left(\frac{4 l^{2}}{\alpha}+2 \delta^{2} \alpha\right) \cdot R_{0}^{2}\right)$. Then the proof is complete.

Lemma 3.5. Under the assumptions (1.2)-(1.7), for $\rho_{2}>0$ in Lemma 3.4, there exists $R_{1} \geq \max \left\{\rho_{2}, \frac{2 \rho_{1}}{\alpha}\right\}$, such that

$$
\begin{aligned}
& \sup _{z \in \mathrm{B}_{\tau}\left(R_{0}\right)}\left\{\varepsilon(t)\left\|A^{\frac{1}{2}} v\right\|^{2}+\|A u\|^{2}+\int_{\tau}^{\infty}\left\|A^{\frac{1}{2}} v(y)\right\|^{2} d y\right\} \\
& \leq R_{1}, \forall t \in \square .
\end{aligned}
$$

Proof Integrating (3.10) on $[\tau, t]$ with $\delta=0$, we get $\int_{\tau}^{\infty}\left\|A^{\frac{1}{2}} v(y)\right\|^{2} d y \leq \frac{2}{\alpha} \cdot \rho_{1}>0$. Then together with Lemma 3.4 we conclude that (3.11) is true.

Lemma 3.6. Under the assumptions (1.2)-(1.7), $g \in L^{2}\left(\square^{3}\right)$, for any ̀̀ $>0$, there exist $T_{1}=T_{1}(\grave{\mathrm{o}})$, as $t \geq T_{1}$ and $K=K(\grave{\mathrm{o}})>0$, such that

$$
\int_{\Omega_{k}^{c}}\left(\varepsilon(t)|v|^{2}+|\nabla u|^{2}+|u|^{2}\right) d x \leq C \grave{\mathrm{o}}, \forall \mathrm{t} \geq T_{1}, k \geq K(\grave{\mathrm{o}}),
$$

where $\Omega_{k}^{c}=\left\{x \in \square^{n}:|x| \geq k\right\}, C$ is a positive constant.

Proof Choosing a smooth function $\theta$ such that $0 \leq \theta(s) \leq 1$, for any $s \in \square^{+}$, and

$$
\theta(s)=0 \text { for } 0 \leq s \leq 1, \theta(s)=1, \text { for } s \geq 2 .
$$

Then there exists a positive constant $\tilde{C}_{0}$, such that $\max \left\{\left|\theta^{\prime}(s)\right|,\left|\theta^{\prime \prime}(\theta)\right|\right\} \leq \tilde{C}_{0}$ for any $s \in \square^{+}$.

Multiplying (1.1) by $\theta\left(\frac{|x|^{2}}{k^{2}}\right) v$ and integrating over $\square^{3}$, we obtain

$$
\begin{aligned}
& \frac{d}{d t}\left[\int_{\square_{n}} \theta\left(\frac{|x|^{2}}{k^{2}}\right) \cdot\left(\varepsilon(t)|v|^{2}+\lambda|u|^{2}\right) d x\right]+(2(\alpha-\delta \varepsilon(t)) \\
& \left.-\varepsilon^{\prime}(t)\right) \int_{\square^{n}} \theta\left(\frac{|x|^{2}}{k^{2}}\right) \cdot \varepsilon^{\prime}(t)|v|^{2} d x-2 \delta(\alpha-\delta \varepsilon(t)) \\
& \int_{\square_{n}} \theta\left(\frac{|x|^{2}}{k^{2}}\right) \cdot u \cdot v d x-2 \int_{\square^{n}} \theta\left(\frac{|x|^{2}}{k^{2}}\right) \Delta u \cdot v d x \\
& +2 \delta \lambda \int_{\square^{n}} \theta\left(\frac{|x|^{2}}{k^{2}}\right)|u|^{2} d x \\
& =-2 \int_{\square^{n}} \theta\left(\frac{|x|^{2}}{k^{2}}\right) f(x, u) v d x+2 \int_{\square^{n}} \theta\left(\frac{|x|^{2}}{k^{2}}\right) g(x) v d x .
\end{aligned}
$$

Now we deal with each term in the above equation:

First we have 
$-2 \int_{\square n} \theta\left(\frac{|x|^{2}}{k^{2}}\right) \Delta u \cdot v d x$

$=\frac{d}{d t} \int_{\square^{n}} \theta\left(\frac{|x|^{2}}{k^{2}}\right)|\nabla u|^{2} d x+2 \delta \int_{\square^{n}} \theta\left(\frac{|x|^{2}}{k^{2}}\right)|\nabla u|^{2} d x$

$+2 \int_{\square^{n}} \frac{2|x|}{k^{2}} \nabla u \cdot v \cdot \theta^{\prime}\left(\frac{|x|^{2}}{k^{2}}\right) d x \geq \frac{d}{d t} \int_{\square^{n}} \theta\left(\frac{|x|^{2}}{k^{2}}\right)|\nabla u|^{2} d x$

$+2 \delta \int_{\square^{n}} \theta\left(\frac{|x|^{2}}{k^{2}}\right)|\nabla u|^{2} d x-\frac{4 \sqrt{2} \tilde{C}_{0}}{k} \int_{k<|x|<\sqrt{2} k}|\nabla u| \cdot|v| d x$

$\geq \frac{d}{d t} \int_{\square^{n}} \theta\left(\frac{|x|^{2}}{k^{2}}\right)|\nabla u|^{2} d x+2 \delta \int_{\square n} \theta\left(\frac{|x|^{2}}{k^{2}}\right)|\nabla u|^{2} d x$

$-\frac{2 \sqrt{2} \tilde{C}_{0}}{k}\left[\int_{\square_{n}}|\nabla u|^{2} d x+\int_{\square_{n}}|v|^{2} d x\right]$

$\geq \frac{d}{d t} \int_{\square n} \theta\left(\frac{|x|^{2}}{k^{2}}\right)|\nabla u|^{2} d x+2 \delta \int_{\square n} \theta\left(\frac{|x|^{2}}{k^{2}}\right)|\nabla u|^{2} d x$ $-\frac{2 \sqrt{2} \epsilon_{0}}{k} R_{0}^{2}-\frac{2 \sqrt{2} \tilde{C}_{0}}{k}\|\|^{2}$,

and

$$
\begin{aligned}
& \left|2 \delta(\alpha-\delta \varepsilon(t)) \int_{\square^{n}} \theta\left(\frac{|x|^{2}}{k^{2}}\right) \cdot u v d x\right| \\
& \leq 2 \delta \alpha \int_{\square^{n}} \theta\left(\frac{|x|^{2}}{k^{2}}\right)|u| \cdot|v| d x \\
& \leq \frac{\alpha}{2} \int_{\square^{n}} \theta\left(\frac{|x|^{2}}{k^{2}}\right)|v|^{2} d x+2 \delta^{2} \alpha \int_{\square^{n}} \theta\left(\frac{|x|^{2}}{k^{2}}\right)|u|^{2} d x .
\end{aligned}
$$

Moreover, we have that

$$
\begin{aligned}
& 2 \int_{\square^{n}} \theta\left(\frac{|x|^{2}}{k^{2}}\right) f(x, u) v d x \\
& =2 \frac{d}{d t} \int_{\square^{n}} \theta\left(\frac{|x|^{2}}{k^{2}}\right) F(x, u) d x+2 \delta \int_{\square^{n}} \theta\left(\frac{|x|^{2}}{k^{2}}\right) f(x, u) u d x,
\end{aligned}
$$

and

$$
\begin{aligned}
& 2\left|\int_{\square^{n}} \theta\left(\frac{|x|^{2}}{k^{2}}\right) f(x, u) v d x\right| \\
& \leq \frac{\alpha}{2} \int_{\square^{n}} \theta\left(\frac{|x|^{2}}{k^{2}}\right)|v|^{2}+\frac{2}{\alpha} \int_{\square^{n}} \theta\left(\frac{|x|^{2}}{k^{2}}\right)|g|^{2} d x .
\end{aligned}
$$

Combining with the above estimates, we obtain

$\frac{d}{d t}\left[\int_{\square n} \theta\left(\frac{|x|^{2}}{k^{2}}\right)\left(|\nabla u|^{2}+\lambda|u|^{2}+\varepsilon(t)|v|^{2}+2 F(x, u)\right) d x\right]$

$+\delta \int_{\square^{n}} \theta\left(\frac{|x|^{2}}{k^{2}}\right)\left(|\nabla u|^{2}+\lambda|u|^{2}+\varepsilon(t)|v|^{2}+2 \delta f(x, u) u\right) d x$

$\leq \frac{2 \sqrt{2} \tilde{C}_{0}}{k} R_{0}^{2}+2 \delta^{2} \alpha \int_{\square^{n}} \theta\left(\frac{|x|^{2}}{k^{2}}\right)|u|^{2} d x+\frac{2}{\alpha} \int_{\square^{n}} \theta\left(\frac{|x|^{2}}{k^{2}}\right)|g|^{2} d x$ $+\frac{2 \sqrt{2} \tilde{C}_{0}}{k}\|\nabla v\|^{2}$.

Let $k_{1}(\grave{\mathrm{o}})>0$, and any $0<\grave{\mathrm{o}}<1$, such that if $k \geq k_{1}(\mathrm{o})$, then

$$
\frac{2 \sqrt{2} C_{0}}{k} R_{0}^{2}<\grave{\mathrm{o}}
$$

holds, and there there exist $k_{2}(\grave{\mathrm{o}})>0$, such that if $k \geq k_{2}(\grave{\mathrm{o}})$, we have

$$
C \int_{\square^{n}} \theta\left(\frac{|x|^{2}}{k^{2}}\right)|u|^{2} d x<\text { ò. }
$$

since $g \in H_{0}^{1}\left(\square^{n}\right)$, so there exist $k_{3}(\grave{\mathrm{o}})>0$, such that

$$
\frac{2}{\alpha} \int_{\square n} \theta\left(\frac{|x|^{2}}{k^{2}}\right)|g|^{2} d x<\grave{\mathrm{o}},
$$

hold for any $k \geq k_{3}(\grave{\mathrm{o}})$.

So there exist $k_{0}=\max \left\{k_{1}(\grave{\mathrm{o}}), k_{2}(\grave{\mathrm{o}}), k_{3}(\grave{\mathrm{o}})\right\}$, if $k \geq k_{0}$, we infer

$$
\begin{aligned}
& \frac{d}{d t}\left[\int_{\square n} \theta\left(\frac{|x|^{2}}{k^{2}}\right)\left(|\nabla u|^{2}+\lambda|u|^{2}+\varepsilon(t)|v|^{2}\right) d x\right]+ \\
& \delta\left[\int_{\square n} \theta\left(\frac{|x|^{2}}{k^{2}}\right)\left(|\nabla u|^{2}+\lambda|u|^{2}+\varepsilon(t)|v|^{2}\right) d x\right] \\
& \leq 3 \grave{\mathrm{o}}+\frac{2 \sqrt{2} \tilde{C}_{0}}{k}\|\nabla\| \|^{2} .
\end{aligned}
$$

By the Gronwall Lemma on $[\tau, t]$, and Lemma 3.3, Lemma3.5, we can get

$$
\begin{aligned}
& \int_{\square^{n}} \theta\left(\frac{|x|^{2}}{k^{2}}\right)\left(|\nabla u|^{2}+\lambda|u|^{2}+\varepsilon(t)|v|^{2}\right) d x \\
& \leq e^{-\delta(t-\tau)} \int_{\square^{n}} \theta\left(\frac{|x|^{2}}{k^{2}}\right)\left(\left|\nabla u_{\tau}\right|^{2}+\lambda\left|u_{\tau}\right|^{2}+\varepsilon(t)\left|v_{\tau}\right|^{2}\right) d x \\
& +\frac{3 \grave{o}}{v}+\frac{2 \sqrt{2} \tilde{C}_{0}}{k} \int_{\tau}^{t} e^{-\delta\left(t-s\|\nabla\|^{2}\right.} d s \\
& \leq \rho_{0} e^{-\delta(t-\tau)}+\frac{3 \grave{o}}{v}+\frac{2 \sqrt{2} \tilde{C}_{0}}{k} R_{0}^{2},
\end{aligned}
$$

so for given ò $>0$ above, setting $K=K$ (ò) there exist $T_{1}=T_{1}(\grave{\mathrm{o}})$, as $t \geq T_{1}$ and $k \geq K(\grave{\mathrm{o}})$, we have

$$
\int_{\square n} \theta\left(\frac{|x|^{2}}{k^{2}}\right)\left(|\nabla u|^{2}+\lambda|u|^{2}+\varepsilon(t)|v|^{2}\right) d x \leq \frac{6 \grave{o}}{v} .
$$

Then we obtain

$$
\int_{\Omega_{k}^{c}}\left(|\nabla u|^{2}+|u|^{2}+\varepsilon(t)|v|^{2}\right) d x \leq \frac{6 \grave{o}}{\hat{\sigma} v}=C \grave{\mathrm{o}},
$$

where $\hat{\sigma}=\min \{1, \lambda\}$.

4 Existence of the time-dependent global attractor on $\mathrm{H}_{t}$

Theorem 4.1. Under the conditions(1.2)-(1.7), the process $U(t, \tau): \mathrm{H}_{\tau} \rightarrow \mathrm{H}_{t}$ generated by problem (1.1) has a invariant time-dependent global attractor $\mathrm{A}=\left\{A_{t}\right\}_{t \in \square}$ in $H^{1}\left(\square^{3}\right) \times L^{2}\left(\square^{3}\right)$.

In the following, we will obtain the existence of the time-dependent attractor for system (1.1) by using the method of asymptotic contractive function.

Lemma 4.2. Under the assumptions(1.2)-(1.7), $g \in H^{1}\left(\square^{3}\right),\left(u_{n}, u_{n_{t}}\right)$ be the solution corresponding to initial data $\left(u_{0}^{n}, v_{0}^{n}\right) \in B_{T}$ for the problem (1.1). Then for any $k>0$ and $T$ (ò) $>0$ be given, let

$$
\Omega_{k}=\left\{x \in \square^{3}:|x|<k\right\},
$$

we can have:

(i) $u_{n} \rightarrow u^{*}-$ weakly in $L^{\infty}\left(T, t ; L^{6}\left(\Omega_{k}\right)\right)$;

(ii) $u_{n_{t}} \rightarrow u_{t}^{*}-$ weakly in $L^{\infty}\left(T, t ; L^{2}\left(\Omega_{k}\right)\right)$; 
(iii) $u_{n} \rightarrow u$ strong in $L^{2}\left(T, t ; L^{2}\left(\Omega_{k}\right)\right)$;

(iv) $u_{n}(T) \rightarrow u(T)$ and $u_{n}(t) \rightarrow u(t)$ strong in $L^{2}\left(\Omega_{k}\right), L^{4}\left(\Omega_{k}\right)$.

Proof From (4.7), (4.11), $\left\|u_{n}\right\|_{2}^{2}+\varepsilon(\xi)\left\|u_{n_{t}}\right\|^{2}$ is bounded, where the bound depends on the $T$, furthermore, $\left\|u_{n}\right\|_{2}^{2}$ is bounded. Moreover, by (1.2) for fixed $T, \xi \in[T, t], \varepsilon(\xi)$ is bounded, hencell $u_{n_{t}} \|^{2}$ is bounded. Then according to Alaoglu Theorem, and use the continuous emdedding $H_{0}^{1}\left(\Omega_{k}\right) \hookrightarrow L^{6}\left(\Omega_{k}\right)$, compact embedding $H_{0}^{1}\left(\Omega_{k}\right)$ $\hookrightarrow L^{2}\left(\Omega_{k}\right), H_{0}^{1}\left(\Omega_{k}\right) \hookrightarrow L^{4}\left(\Omega_{k}\right)$, the result can be obtained. $\square$

\subsection{A priori estimates}

Let $\left(u_{i}(t), u_{i_{t}}(t)\right)$ be the corresponding solution of (1.1) with initial datum $\left(u_{0}^{i}(\tau), v_{0}^{i}(\tau)\right) \in\left\{B_{\tau}\right\}_{\tau \in \square}$, and

$$
w=u_{1}(t)-u_{2}(t),
$$

then $w(t)$ satisfies

$$
\left\{\begin{array}{l}
\varepsilon(t) \omega_{t t}+\alpha \omega_{t t}-\square \omega+\lambda \omega+f\left(x, u_{1}\right)-f\left(x, u_{2}\right)=0, t>T, \\
\omega(x, T)=u_{0}^{1}(T)-u_{0}^{2}(T), \omega_{t}(x, T)=v_{0}^{1}(T)-v_{0}^{2}(T),
\end{array}\right.
$$

Denote

$$
\left.\mathrm{E}_{w}(t)=\frac{1}{2}\|\nabla u\|^{2}+\lambda\|w\|^{2}+\varepsilon(t)\left\|w_{t}\right\|^{2}\right] .
$$

Taking the inner product (4.1) with $w_{t}$ in $L^{2}\left(\square^{3}\right)$ we find $\frac{d}{d t} \mathrm{E}_{w}(t)+\left(\alpha-\frac{\varepsilon^{\prime}(t)}{2}\right)\left\|w_{t}\right\|^{2}+\left\langle f\left(x, u_{1}\right)-f\left(x, u_{2}\right), w_{t}(\xi)\right\rangle=0$.

Integrating (4.2) over $[s, t]$, we have

$$
\begin{aligned}
& \mathrm{E}_{w}(t)-\mathrm{E}_{w}(s)+\int_{s}^{t}\left(\alpha-\frac{\varepsilon^{\prime}(\xi)}{2}\right)\left\|w_{t}(\xi)\right\|^{2} d \xi \\
& +\int_{s}^{t}\left\langle f\left(x, u_{1}\right)-f\left(x, u_{2}\right), w_{t}(\xi)\right\rangle d \xi=0,
\end{aligned}
$$

where $T \leq s \leq t$. For $L<2 \alpha$, where $L$ is the bound of $\varepsilon(t), \varepsilon^{\prime}(t)$, then we can get

$\int_{T}^{t} \varepsilon(\xi)\left\|\omega_{t}(\xi)\right\|^{2} d \xi \leq \mathrm{E}_{\omega}(T)-\int_{T}^{t}\left\langle f\left(x, u_{1}\right)-f\left(x, u_{2}\right), \omega_{t}(\xi)\right\rangle d s$.

Then multiplying (4.1) by $\omega$, and integrating over $\square^{3} \times[T, t]$, we obtain

$$
\begin{aligned}
& \left.\int_{T}^{t}\|\nabla \omega(s)\|^{2}+\lambda\|\omega(s)\|^{2}\right) d s+\varepsilon(t)\left\langle\omega_{t}(t), \omega(t)\right\rangle \\
& =\left\langle\varepsilon(T) \omega_{t}(T), \omega(T)\right\rangle-\int_{T}^{t}\left(\alpha-\varepsilon^{\prime}(s)\right)\left\langle\omega_{t}(s), \omega(s)\right\rangle d s \\
& +\int_{T}^{t} \varepsilon(s)\left\|\omega_{t}(s)\right\|^{2} d s-\int_{T}^{t}\left\langle f\left(x, u_{1}\right)-f\left(x, u_{2}\right), \omega(s)\right\rangle d s .
\end{aligned}
$$

Therefore, from (4.4) and (4.5), yields

$$
\begin{aligned}
& 2 \int_{T}^{t} \mathrm{E}_{\omega}(s) \leq 2 \mathrm{E}_{\omega}(T)-2 \int_{T}^{t}\left\langle f\left(x, u_{1}\right)-f\left(x, u_{2}\right), \omega_{t}(s)\right\rangle d s \\
& -\int_{T}^{t}\left(\alpha-\varepsilon^{\prime}(s)\right)\left\langle\omega_{t}(s), \omega(s)\right\rangle d s-\varepsilon(t)\left\langle\omega_{t}(t), \omega(t)\right\rangle \\
& +\varepsilon(T)\left\langle\omega_{t}(T), \omega(T)\right\rangle-\int_{T}^{t}\left\langle f\left(x, u_{1}\right)-f\left(x, u_{2}\right), \omega(s)\right\rangle d s
\end{aligned}
$$

Integrating (4.3) over $[T, t]$, we have

$$
\begin{aligned}
& (t-T) \mathrm{E}_{\omega}(t)+\int_{T}^{t} \int_{s}^{t}\left(\alpha-\frac{\varepsilon^{\prime}(\xi)}{2}\right)\left\|\omega_{t}(\xi)\right\|^{2} d \xi d s \\
& =-\int_{T}^{t} \int_{s}^{t}\left\langle f\left(x, u_{1}(\xi)\right)-f\left(x, u_{2}(\xi)\right), \omega_{t}(\xi)\right\rangle d \xi d s+\int_{T}^{t} \mathrm{E}_{\omega}(s) d s,
\end{aligned}
$$

together with (4.6), there holds

$$
\begin{aligned}
& (t-T) \mathrm{E}_{\omega}(t) \leq \mathrm{E}_{\omega}(T)+\frac{1}{2}\left\langle\varepsilon(T) \omega_{t}(T), \omega(T)\right\rangle \\
& -\frac{1}{2}\left\langle\varepsilon(t) \omega_{t}(t), \omega(t)\right\rangle-\frac{1}{2} \int_{T}^{t}\left(\alpha-\varepsilon^{\prime}(s)\right)\left\langle\omega_{t}(s), \omega(s)\right\rangle d s \\
& -\frac{1}{2} \int_{T}^{t}\left\langle f\left(x, u_{1}\right)-f\left(x, u_{2}\right), \omega(s)\right\rangle d s \\
& -\int_{T}^{t}\left\langle f\left(x, u_{1}\right)-f\left(x, u_{2}\right), \omega_{t}(s)\right\rangle d s \\
& -\int_{T}^{t} \int_{s}^{t}\left\langle f\left(x, u_{1}(\xi)\right)-f\left(x, u_{2}(\xi)\right), \omega_{t}(\xi)\right\rangle d \xi d s .
\end{aligned}
$$

Set

$\psi_{T}^{t}\left(\left(u_{0}^{1}(T), v_{0}^{1}(T)\right),\left(u_{0}^{2}(T), v_{0}^{2}(T)\right)\right)$

$=-\frac{1}{2(t-T)}\left\langle\varepsilon(t) \omega_{t}, \omega\right\rangle-\frac{1}{2(t-T)} \int_{T}^{t}\left(\alpha-\varepsilon^{\prime}(s)\right)\left\langle\omega_{t}(s), \omega(s)\right\rangle d s$

$-\frac{1}{2(t-T)} \int_{T}^{t}\left\langle f\left(x, u_{1}\right)-f\left(x, u_{2}\right), \omega(s)\right\rangle d s$

$-\frac{1}{t-T} \int_{T}^{t}\left\langle f\left(x, u_{1}\right)-f\left(x, u_{2}\right), \omega_{t}(s)\right\rangle d s$

$-\frac{1}{t-T} \int_{T}^{t} \int_{s}^{t}\left\langle f\left(x, u_{1}(\xi)\right)-f\left(x, u_{2}(\xi)\right), \omega_{t}(\xi)\right\rangle d \xi d s$,

and

$$
C_{M}=\mathrm{E}_{\omega}(T)+\frac{1}{2}\left\langle\varepsilon(T) \omega_{t}(T), \omega(T)\right\rangle,
$$

then we have

$$
\mathrm{E}_{\omega}(t) \leq \frac{C_{M}}{t-T}+\psi_{T}^{t}\left(\left(u_{0}^{1}(T), v_{0}^{1}(T)\right),\left(u_{0}^{2}(T), v_{0}^{2}(T)\right)\right) .
$$

\subsection{Asymptotically compact}

Theorem 4.3. Under the assumption (1.2) - (1.7), then the process $\{U(t, \tau)\}$ is a asymptotic contractive process, that is, for any fixed $t \in \square$, bounded sequence $\left\{x_{n}\right\}_{n=1}^{\infty} \subset X_{\tau_{n}}$ and any $\left\{\tau_{n}\right\}_{n=1}^{\infty} \subset \square^{-t}$, with $\tau_{n} \rightarrow-\infty$ as $n \rightarrow \infty$, sequence $\left\{U\left(t, \tau_{n}\right) x_{n}\right\}_{n=1}^{\infty}$ is precompact in $H^{1}\left(\square^{n}\right) \times L^{2}\left(\square^{n}\right)$.

Proof Let

$\psi_{T}^{t}\left(\left(u_{0}^{1}(T), v_{0}^{1}(T)\right),\left(u_{0}^{2}(T), v_{0}^{2}(T)\right)\right)=-\frac{1}{2(t-T)} \int_{\Omega_{k}} \varepsilon(t) \omega_{t} \cdot \omega d x$

$-\frac{1}{2(t-T)} \int_{T}^{t}\left(\alpha-\varepsilon^{\prime}(s)\right) \int_{\Omega_{k}} \omega_{t}(s) \cdot \omega(s) d x d s$

$-\frac{1}{2(t-T)} \int_{T}^{t} \int_{\Omega_{k}}\left(f\left(x, u_{1}\right)-f\left(x, u_{2}\right)\right) \cdot \omega(s) d x d s$

$-\frac{1}{t-T} \int_{T}^{t} \int_{\Omega_{k}}\left(f\left(x, u_{1}\right)-f\left(x, u_{2}\right)\right) \cdot \omega_{t}(s) d x d s$

$-\frac{1}{t-T} \int_{T}^{t} \int_{s}^{t} \int_{\Omega_{k}}\left(f\left(x, u_{1}(\xi)\right)-f\left(x, u_{2}(\xi)\right)\right) \cdot \omega_{t}(\xi) d x d \xi d s$.

From Lemma 3.6, as $m, n$ large enough, and by Hölder, Young inequalities, we can have 


$$
\begin{aligned}
& \int_{\Omega_{k}^{c}} \varepsilon(t)\left(u_{n_{t}}(t)-u_{m_{t}}(t)\right)\left(u_{n}(t)-u_{m}(t)\right) d x \\
& \leq L\left\|u_{n_{t}}(t)-u_{m_{t}}(t)\right\|_{L^{2}\left(\Omega_{k}^{c}\right)}\left\|u_{n}(t)-u_{m}(t)\right\|_{L^{2}\left(\Omega_{k}^{c}\right)} \leq \frac{\grave{\mathrm{o}}}{4},
\end{aligned}
$$

and

$\int_{T}^{t} \int_{\Omega_{k}^{c}}\left(u_{n_{t}}(s)-u_{m_{t}}(s)\right)\left(u_{n}(s)-u_{m}(s)\right) d x d s$

$\leq \sup _{T \leq s \leq t}\left\|u_{n_{t}}(s)-u_{m_{t}}(s)\right\|_{L^{2}\left(\Omega_{k}^{c}\right)}\left\|u_{n}(s)-u_{m}(s)\right\|_{L^{2}\left(\Omega_{k}^{c}\right)} \leq \frac{\mathrm{o}}{4}$.

Similar as $m, n$ large enough

$\int_{T}^{t} \int_{\Omega_{k}^{c}}\left(f\left(x, u_{n}\right)-f\left(x, u_{m}\right)\right)\left(u_{n_{t}}(s)-u_{m_{t}}(s)\right) d x d s$

$\leq l \sup _{T \leq s \leq t}\left\|u_{n_{t}}(s)-u_{m_{t}}(s)\right\|_{L^{2}\left(\Omega_{k}^{c}\right)}\left\|u_{n}(s)-u_{m}(s)\right\|_{L^{2}\left(\Omega_{k}^{c}\right)} \leq \frac{\grave{\mathrm{o}}}{4}$.

Also we can obtain that as $m, n$ large enough $\int_{T}^{t} \int_{s}^{t} \int_{\Omega_{k}^{c}}\left(u_{n_{t}}(\xi)-u_{m_{t}}(\xi)\right)\left(f\left(x, u_{n}(\xi)\right)-f\left(x, u_{m}(\xi)\right)\right) d x d \xi d s$ $\leq \frac{\grave{\mathrm{o}}}{4}$.

So we can get

$$
\begin{aligned}
& \psi_{T}^{t}\left(\left(u_{n}^{1}(T), v_{n}^{1}(T)\right),\left(u_{m}^{2}(T), v_{m}^{2}(T)\right)\right) \\
& \leq \grave{\mathrm{o}}+\phi_{T}^{t}\left(\left(u_{n}^{1}(T), v_{n}^{1}(T)\right),\left(u_{m}^{2}(T), v_{m}^{2}(T)\right)\right)
\end{aligned}
$$

Next, for any fixed ò $>0$, and some fixed $t, \operatorname{let} T<t$ such that $t-T$ so large that

$$
\frac{C_{M}}{t-T}<\frac{\grave{o}}{2} .
$$

Hence, from definition $3.1,3.2$, we only need to verify that $\phi_{T}^{t}$ in (4.17) is the contractive function for each fixed $T$.

Now, we will deal with each term in (4.12) one by one.

Firstly, from Lemma 3.2 and (i)-(iv) in Lemma 4.1, we get

$$
\lim _{n \rightarrow \infty} \lim _{m \rightarrow \infty} \int_{\Omega_{k}} \varepsilon(t)\left(u_{n_{t}}(t)-u_{m_{t}}(t)\right)\left(u_{n}(t)-u_{m}(t)\right) d x=0,
$$

$\lim _{n \rightarrow \infty} \lim _{m \rightarrow \infty} \int_{T}^{t} \int_{\Omega_{k}} L\left(u_{n_{t}}(s)-u_{m_{t}}(s)\right)\left(u_{n}(s)-u_{m}(s)\right) d x d s=0$,

$\lim _{n \rightarrow \infty} \lim _{m \rightarrow \infty} \int_{T}^{t} \int_{\Omega_{k}}\left(f\left(x, u_{n}\right)-f\left(x, u_{m}\right)\right)\left(u_{n}(s)-u_{m}(s)\right) d x d s=0$.

Similar to $[4,16]$, we have

$$
\lim _{n \rightarrow \infty} \lim _{m \rightarrow \infty} \int_{T}^{t} \int_{\Omega_{k}}\left(f\left(x, u_{n}\right)-f\left(x, u_{m}\right)\right)\left(u_{n_{t}}(s)-u_{m_{t}}(s)\right) d x d s=0,
$$

At the same time, for each fixed $t$, $\left|\int_{s}^{t} \int_{\Omega_{k}}\left(u_{n_{t}}(\xi)-u_{m_{t}}(\xi)\right)\left(f\left(x, u_{n}(\xi)\right)-f\left(x, u_{m}(\xi)\right)\right) d x d \xi\right|$

bounded, then by the Lebesgue dominated convergence theorem we have

$\lim _{n \rightarrow \infty} \lim _{m \rightarrow \infty} \int_{T}^{t} \int_{s}^{t} \int_{\Omega_{k}}\left(u_{n_{t}}(\xi)-u_{m_{t}}(\xi)\right)\left(f\left(x, u_{n}(\xi)\right)-f\left(x, u_{m}(\xi)\right)\right) d x d \xi d s$

$=\int_{T}^{t}\left(\lim _{n \rightarrow \infty} \lim _{m \rightarrow \infty} \int_{s}^{t} \int_{\Omega_{k}}\left(u_{n_{t}}(\xi)-u_{m_{t}}(\xi)\right)\left(f\left(x, u_{n}(\xi)\right)-f\left(x, u_{m}(\xi)\right)\right) d x d \xi\right) d s$ $=0$.
Hence, collecting all (4.18)-(4.21), we get that $\phi_{T}^{t}$ is the contractive function, so $\phi_{T}^{t}$ is asymptotic contractive function, then from (4.21) we know that the process is asymptotical contractive process.

Proof of Theorem 4.1 From Lemma 3.3-Lemma 3.6, and Theorem 4.3,Theorem 2.9 we can easily obtain the result.

\section{Acknowledgements}

The authors would like to thank the reviewers and the editors for their valuable suggestions and comments.

\section{Funding}

This work was supported by the NSF of China (11961059).

\section{Availability of data and materials}

Not applicable.

\section{Competing interests}

The authors declare that they have no competing interests.

\section{Authors contributions}

All authors contributed equally to the writing of this paper. All authors read and approved the final manuscript.

\section{REFERENCES}

[1] M. Conti, V. Pata, R. Temam, Attractors for processes on time-dependent spaces. Applications to wave equations, J. Differ. Equ, 255 (2013) 1254-1277.

[2] M. Conti, V. Pata, Asymptotic structure of the attractor for processes on time-dependentspaces, Nonl. Anal. RWA 19 (2014) 1-10.

[3] M. Conti, V. Pata, On the time-dependent Cattaneo law in space dimension one, Appl. Math. Comput. 259 (2015) 32-44.

[4] Q.Z. Ma, J. Wang, T.T. Liu, Time-dependent asymptotic behavior of the solution for wave equations with linear memory, Comput. Appl. Math 76 (2018) 1372-1387.

[5] D. Plinio, G. S. Duane, R. Temam, Time-Dependent attractor for the oscillon equation, Discrete Contin. Dyn. Syst. 29 (2011) 141-167.

[6] F.J. Meng, M.H. Yang, C.K. Zhong, Attractors for wave equations with nonlinear damping on timedependent space, Discrete Contin. Dyn. Syst. Ser. B 1 (2016) 205-225.

[7] F.J. Meng, C.C. Liu, Necessary and sufficient conditions for the existence of time-dependent global attractor and application, J. Math. Phys. 58 (2017) 1-9.

[8] A.V. Babin, M.I. Vishik, Attractors of partial differential evolution equation in an unbounded domain, Proc R Soc Edinburgh 116A (1990) 221-243.

[9] N.I. Karachalios, N.M. Stavrakakis, Existence of a global attractor for semilinear dissipative wave equation on $\square^{N}$, J. Differ. Equ, 157(1999) 183-205.

[10]V. Pata, Attractors for a damped wave equation on $\square^{3}$ with linear memory, Math. Methods Appl. Sci. 23(2000) 633-653. 
[11]Z.J. Yang, P.Y. Ding, Longtime dynamics of the Kirchhoff equation with strong damping and critical nonlinearity on $\square^{N}$, J. Math. Anal. Appl. 434 (2016) 1826-1851.

[12]A.V. Babin, M.I. Vishik, Attractors of Evolution Equations, North-Holland, Amsterdam, 1992.

[13]C.Y. Sun, D.M. Cao, J.Q. Duan, Non-autonomous dynamics of wave equations with nonlinear damping and critical nonlinearity, Nonlinearity 19 (2006) 2645-2665.

[14]C. Y. Sun, M. H. Yang, C. K. Zhong, Global attractors for the wave equation with nonlinear damping, J. Differ. Equ, 227 (2006), 427-423.

[15]C. Y. Sun, D. M. Cao, J. Q. Duan, Uniform attractors for nonautonomous wave equations with nonlinear damping, SIAM J. Appl. Dyn. Syst., 6 (2007), 293-318.

[16]J.C. Robinson, Infinite-Dimensional Dynamical Systems, Cambridge University Press, NewYork, 2001

[17]Q. F. Ma, S. H. Wang and C. K. Zhong, Necessary and sufficient conditions for the existence of global attractor for semigroups and applications, Indiana Univ. Math. J., 51 (2002), 1541-1559.

[18]T. T. Liu, Q. Z. Ma, Time-dependent attractor for plate equations on, $\square^{n}$ J. Math. Anal. Appl., 479 (2019) 315-332. 